\title{
An dieser Schnittstelle treffen sich Bergretter und Klinikärzte
}

\author{
Kürzlich eröffnete die Klinik Immenstadt das erste „Zentrum für Gebirgsmedizin“ in Deutschland. Wir sprachen \\ mit dem Chefarzt der Unfallchirurgie über die Ziele des neuen Zentrums.
}

\section{$?$}

Dr. med. Herbert Mayer Facharzt für Orthopädie, Unfallchirurgie, Spezielle Unfallchirurgie und Notfallmedizin, Zentrum für Gebirgsmedizin, Klinik Immenstadt

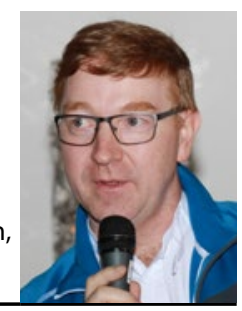

MMW: Warum wurde das Zentrum für Gebirgsmedizin gegründet?

Mayer: Das Zentrum für Gebirgsmedizin soll für eine bessere Verzahnung von Präklinik und Klinik sorgen. Zum einen haben wir festgestellt, dass Bergretter oft noch Fragen zu den Patienten haben, etwa ob alles gut gelaufen ist. Dann wissen sie meist nicht, an wen sie sich wenden können. Umgekehrt würden wir auch den Rettern gern manche Informationen zurückspiegeln. Hier fehlt bislang

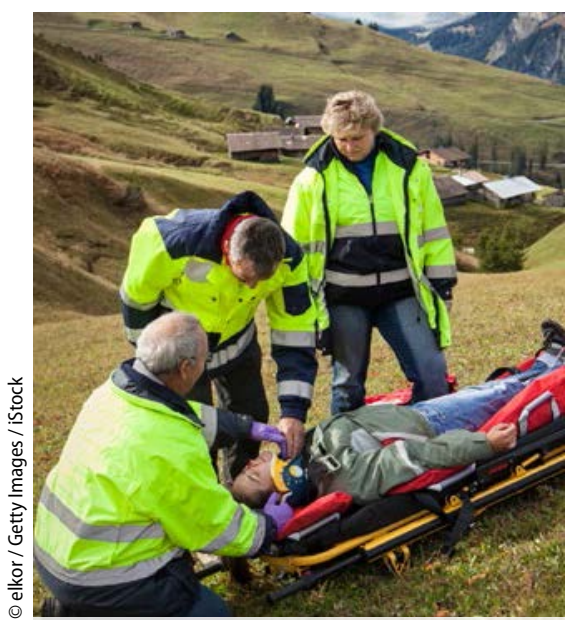

Bergretter brauchen für Not- und Unfälle sichere Algorithmen. eine gemeinsame Kommunikationsplattform. Außerdem wollen wir die strukturierte Fort- und Weiterbildung auf dem alpenmedizinischen Gebiet verbessern. So wollen wir den „Bergrettungstag", den wir gerade zu fachübergreifenden Notfällen im Gebirge veranstaltet haben, dauerhaft etablieren.

MMW: Was ist sonst bei der Bergrettung und der anschließenden Versorgung zu verbessern?

Mayer: Wir brauchen gebirgsspezifische Algorithmen vom Unfallort am Berg bis in die Klinik. Für den Herzinfarkt etwa gibt es Algorithmen in der Straßenrettung, um die Therapie auf ein Minimum $\mathrm{zu}$ reduzieren und den Patienten so schnell wie möglich zum Herzkatheterplatz zu bringen. Im Gebirge funktioniert das aber manchmal nicht, etwa in der Nacht oder wenn bei schlechtem Wetter der Hubschrauber nicht fliegt. Dann kann das nötige Zeitlimit nach dem Infarkt nicht eingehalten werden, und es besteht eine gewisse Unsicherheit, wie verfahren werden soll.

Unsicherheiten gibt es auch bei Erfrierungen. Hier existieren Algorithmen, die präklinisch ein Wiederaufwärmen der Extremität empfehlen - und andere, die das nicht empfehlen. Das hängt auch von den lokalen Gegebenheiten ab. Wir erstellen gerade einen Algorithmus für Erfrierungen speziell in den Allgäuer Bergen. Einen genauen Behandlungspfad wünscht man sich auch zur Frage, ob Sprunggelenksbrüche noch direkt am Berg eingerichtet werden sollten.

\section{MMW: Sie wollen auch präventiv tätig werden und eine Sprechstunde für Kletterer und Bergsteiger einrich- ten. Worum ginge es dabei?}

Mayer: Die Einrichtung der Sprechstunde ist für das kommende Jahr geplant. Es gibt ja immer wieder Fragen wie: „Kann ich mit meiner Endoprothese ins Gebirge?“ oder „Kann ich mit COPD Seilbahn fahren?" Eine solche Beratung planen wir zunächst für normale Bergsportler hier im Allgäu. Langfristig sind aber auch eine umfassendere Reiseberatung und Angebote zu Voruntersuchungen angedacht, etwa für Personen, die nach Nepal möchten und sich zur Höhenkrankheit informieren wollen.

Interview: Dr. Christine Starostzik

\section{Zahl der Bergnotfälle steigt leicht an}

Das Risiko, beim Bergsport tödlich zu verunglücken, sinkt seit mehr als 60 Jahren und befindet sich auf einem historischen Tiefstand. Allerdings nimmt die Zahl alpiner Notfälle insgesamt seit den 1990er-Jahren leicht zu. Das hat der Deutsche Alpenverein (DAV) auf Basis der Meldungen seiner Mitglieder an die vereinseigene Versicherung ermittelt. Laut DAV ist der Anstieg vor allem darauf zurückzuführen, dass immer mehr Menschen in die Berge gehen - und dass im Notfall die Alarmierung mit Handy nahezu reibungslos funktioniert. Einen sichtbaren Effekt habe aber auch die „Übermotivation“, mit der viele Bergsportler an „Prestigebergen“ wie der Zugspitze oder dem Watzmann unterwegs sind. Insgesamt verunglücken dem DAV zufolge v. a. unerfahrene Wanderer und Kletterer - und diejenigen, die nicht die passenden Touren für sich auswählen. 